\title{
Acquired facial lipoatrophy: pathogenesis and therapeutic options
}

\author{
Aneta Szczerkowska-Dobosz ${ }^{1}$, Barbara Olszewska², Małgorzata Lemańska³ ${ }^{3}$ Dorota Purzycka-Bohdan ${ }^{1}$, Roman Nowicki ${ }^{1}$ \\ ${ }^{1}$ Department of Dermatology, Venereology and Allergology, Medical University of Gdansk, Gdansk, Poland \\ Head of the Department: Prof. Roman Nowicki MD, PhD \\ ${ }^{2}$ Students' Scientific Group of Dermatology, Venerology and Allergology Department, Medical University of Gdansk, Gdansk, Poland \\ Head of the Department: Aneta Szczerkowska-Dobosz MD, PhD \\ ${ }^{3}$ Department of Infectious Disease, Medical University of Gdansk, Gdansk, Poland \\ Head of the Department: Tomasz Smiatacz MD, PhD
}

Postep Derm Alergol 2015; XXXII, 2: 127-133

DOI: 10.5114/pdia.2014.40971

\begin{abstract}
Facial lipoatrophy refers to the loss of subcutaneous fat tissue presenting by flattening or indentation of convex contour of the face. Facial lipoatrophy is a feature of the normal ageing process. It may be also a manifestation of chronic diseases, most frequently it affects HIV-infected individuals treated with highly active antiretroviral therapy (HAART) and may constitute a complication of connective tissue diseases, like lupus erythematosus profundus or morphea. Early recognition and treatment of the active stage of connective tissue diseases is of essential significance in prevention of subsequent scarring and atrophy lesions. In HIV-positive patients undergoing HAART therapy, the attempt to modify thetreatment scheme so it has a less lipemic effect seems to be justified. Esthetic correction of facial lipoatrophy in chronic diseases is a great challenge. Improvement of appearance is very important for affected individuals, because it diminishes their stigmatization and psychosocial dysfunction. Facial volumetric correction includes surgical and dermatological procedures such as adipose transfer and injectable dermal fillers.
\end{abstract}

Key words: face lipoatrophy, connective tissue diseases, highly active antiretroviral therapy.

\section{Introduction}

Facial lipoatrophy refers to the loss of adipose tissue and is manifested by flattening or indentation of convex contours of the face. A lipodystrophy is a wider term associated with abnormalities of fat tissue distribution and its metabolism, leading to excessive loss and/or accumulation of adipocytes. The lipoatrophy most frequently affects the subcutaneous fat layer. According to the anatomical localization, it can be classified as generalized, partial or localized [1]. Due to genetic susceptibility, the lipoatrophic disorders are divided into congenital and acquired. The congenital lipoatrophy refers to the rare conditions with a predominantly autosomal recessive pattern of inheritance. These disorders may occur as generalized or partial forms and are usually accompanied by metabolic disturbances, especially hypoleptinemia, hypertriglyceridemia and insulin resistance [2]. The acquired lipoatrophy may affect people in all age groups as a result of repeated trauma, pressure, drug injections or as a consequence of some chronic diseases. Physiological facial lipoatrophy is associated with a normal course of ageing.

The most common locations of adipose tissue loss in the course of facial lipoatrophy are cheeks, temples and the preauricular, orbital or perioral region. With the progress of the disease both some bone structures and muscles may be excessively visible, especially zygomatic arch, orbit, anatomical details of mandible and jaw, zygomaticus major and minor muscles, risorius muscle, levator muscle of the upper lip, orbicularis oculi muscles, masticator muscle, temporal muscles. A 5 degree scale can be applied in clinical practice to establish the degree of lipoatrophy severity. The first degree indicates discrete flattening or indentation of one or more facial regions (cheeks, temple, preauricular, orbital and perioral area), lack of protrusion of bones and invisible facial muscles, whereas the fifth scale degree is linked with excessive in-

Address for correspondence: Aneta Szczerkowska-Dobosz MD, PhD, Department of Dermatology, Venereology and Allergology, Medical University of Gdansk, 7 Debinki St, 80-211 Gdansk, Poland, phone: +48 509093 699, e-mail: adobosz@gumed.edu.pl Received: 3.11.2013, accepted: 6.01.2014. 
dentation of one or more regions of the face with strongly protruding facial bone structures and muscles [3].

The diagnostic techniques such as computed tomography $(\mathrm{CT})$, magnetic resonance imaging (MRI) or ultrasound examination (USG), are not used to measure severity of the lipoatrophy mainly due to the costs and lack of consensus-based standards on the interpretation of the images.

\section{Etiopathogenesis of acquired facial lipoatrophy}

Progressive facial adipose tissue loss constitutes a part of the natural ageing process. A significant decrease in fat volume in cheeks, temples and orbits starts to be visible at the age of 20 , however changes in contour of the face begin to be noticeable roughly at the age of 30 [4]. The fat distribution is one of the decisive factors which have an impact on the appearance of young and old looking face. With the ageing, the adipocyte tissue of the periorbital, frontal, temporal and perioral region and cheeks, undergoes atrophy whereas the hypertrophy is seen in the submandibular region, mandibular arch, nasolabial fold, mentolabial fold and infraorbital area. The facial fat pads also modify their shape and location

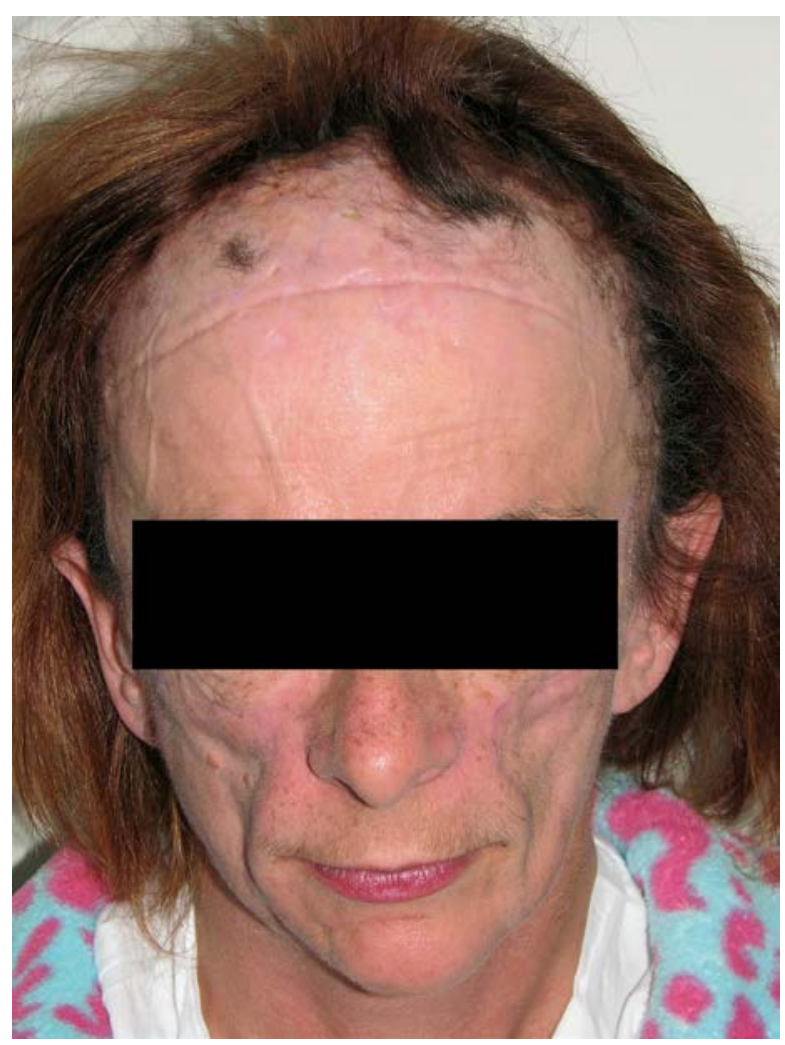

Figure 1. Female with lupus erythematosus profundus. Depression of both cheeks with significant face deformation. Relocation of the hairline due to scarring alopecia in the course of LEP due to facial skeleton changes. As a result of the bone resorption process, orbital entrance areas become wider, the jaw moves back and the frontal process decreases its thickness. The mandibular angle becomes more obtuse and the height and length of its corpus decrease. The loss of bone support in these locations causes the indentation of soft tissues. In consequence, the face loses its full young oval shape and facial contours become more prominent.

In contrast with natural lipoatrophy, which is slow and symmetric, the pathological loss of adipose tissue has a dynamic, progressive course, may be asymmetric and often leads to both physical suffering and psychological ramifications such as own body image disturbances or social withdrawal. Significant and rapid facial fat loss may cause discomfort associated with abnormal skin tension on facial skeleton.

The most common causes of facial lipoatrophy connected with chronic disease are the lipoatrophy due to antiretroviral therapy for HIV infection and the lipoatrophy as a consequence of connective tissue diseases associated with panniculitis.

Panniculitis of the face is a rare manifestation of connective tissue diseases such as lupus erythematosus and localized scleroderma. The lipoatrophy in the course of connective tissue diseases is usually preceded by the inflammatory, painful phase and may cause a deep contour loss of facial tissues. Anatomical structures adjacent to facial fat pads can be also affected. Masticator and mimic muscle involvement by the disease limits their mobility. Moreover, the nerve infiltration with the following neuropathic pain and paresis can lead to deeper loss of natural contours of the face mainly due to nerve damage and muscle fibers atrophy.

The characteristic symptoms of lupus erythematosus profundus (LEP) were first reported in 1883 by Kaposi. In 1956, Arnold described it as a subtype of chronic cutaneous lupus erythematosus [5]. Lupus erythematosus profundus may be an isolated entity or more frequently is associated with discoid lupus erythematosus (DLE) or systemic lupus erythematosus (SLE). The clinical manifestation of the early stage of LEP is characterized by hardened subcutaneous plaques and nodules as a result of deeper dermal layers involvement. Lesions are typically located on the chest, proximal limbs, hips, buttocks, cheeks and may appear uni- or bilaterally [6]. Skin findings are often recurrent and resolve, leaving scarring and atrophy (Figure 1). Histopathological examination of the skin biopsy reveals lobular panniculitis with lymphocytic and plasmocytic infiltration, karyorrhexis and hyaline necrosis of fat lobules. The anti-nuclear antibody (ANA) test is usually positive, but it is not required to establish the diagnosis of LEP. Lupus panniculitis occurs in 2-3\% of patients with SLE. Due to the fact that the diagnosis of LEP may precede the presentation of SLE, patients suffering from LEP should be monitored for symptoms of SLE. 
Localized scleroderma, also known as morphea, is a form of scleroderma limited to the skin and subcutaneous fatty tissue. The internal organ involvement and Reynaud's phenomenon are not usually observed. According to the classification proposed by Peterson et al., the morphea is divided into the following five forms: plaque, generalized, bullous, linear, and deep [6]. In the last two patterns of morphea, lesions can extend over subcutaneous tissues and affect underlying muscles and bones. The first clinical feature of localized scleroderma is an inflammatory erythematous plaque. The lesions become hard and white in the center surrounded by a purplish halo [7]. In linear and deep morphea, collagen fibers reach the subcutaneous fat tissue and, in consequence, the panniculitis, fibrosis and deep atrophy appear (Figure 2). This type of scleroderma usually involves extremities, face and head. En coup de sabre and Parry-Romberg syndrome (Progressive Facial Hemiatrophy) are considered deep forms of linear scleroderma. Parry-Romberg syndrome is characterized by progressive shrinkage and atrophy of the tissues beneath the skin, usually only on one side of the face, without or with minimal skin involvement. Progressive facial asymmetry may lead to serious deformations.

Facial lipoatrophy associated with HIV infection was first described in 1998 as one of the symptoms of the generalized lipodystrophy, developing in patients receiving highly active antiretroviral therapy (HAART) [8]. The pathogenesis of HIV-associated lipodystrophy is probably based on blockage of protein synthesis connected with lipid metabolism by antiretroviral drugs with the subsequent apoptosis and disturbances of peripheral adipocyte differentiation. Thus far, Carr et al. have made a significantcontribution to the progress of knowledge about lipodystrophy pathogenesis in patients with HIV infection, which is still not clearly defined [9]. In addition, the resolution of this problem is being complicated by the introduction of many new antiretroviral medications, as well, as new classes of antiretroviral drugs.

The prevalence of HIV-associated lipodystrophy depends on accepted diagnostic criteria and may reach even $83 \%$ [8, 9]. Risk factors for HIV-associated lipodystrophy in patients treated with HAART include low body weight before therapy, hypertriglyceridemia, increased C-peptide level, low HDL cholesterol level in the first year after treatment, total duration of HIV infection and the long-term anti-HIV therapy with protease inhibitors [9]. Clinical presentation of the lipodystrophy associated with antiretroviral therapy includes the hypertrophy of visceral adipose tissue and abdomen, nape and sometimes mammary gland. The adipose tissue atrophy of extremities, buttocks, cheeks, nasolabial folds, temples and, in the late stage of the disease, the periorbital region is also observed (Figure 3). Changes in fat distribution may be accompanied by metabolic disturbances such as insulin resistance, hypertriglyceridemia, hypercholesterolemia and a low HDL cholesterol level. These metabolic abnor-

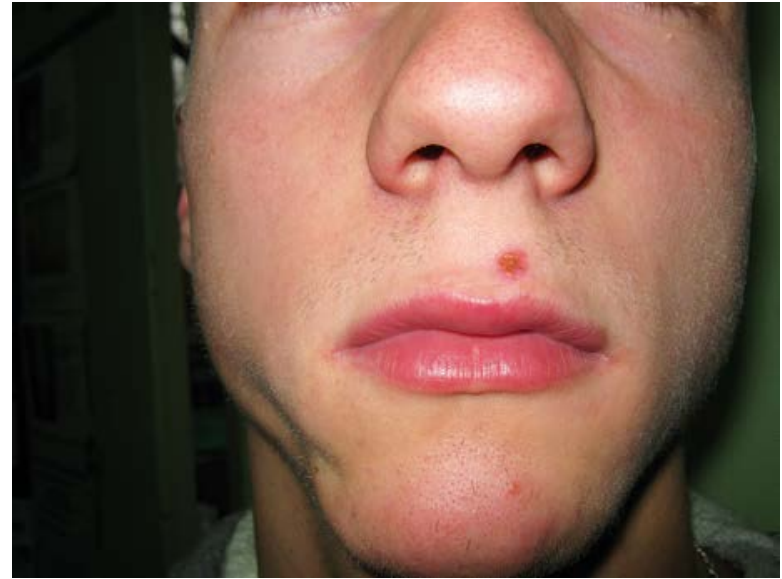

Figure 2. Young male patient with a deep form of morphea affecting the right mandibular region with face contour disturbance

malities may lead to development of type 2 diabetes, atherosclerosis and increase the risk of death due to cardiovascular diseases. Typical clinical presentation, concomitant metabolic disturbances and long-term antiretroviral therapy play a key role in differentiation between the lipodystrophy and the cachexia in the late stage of AIDS, with the muscle mass loss as a major manifestation [10].

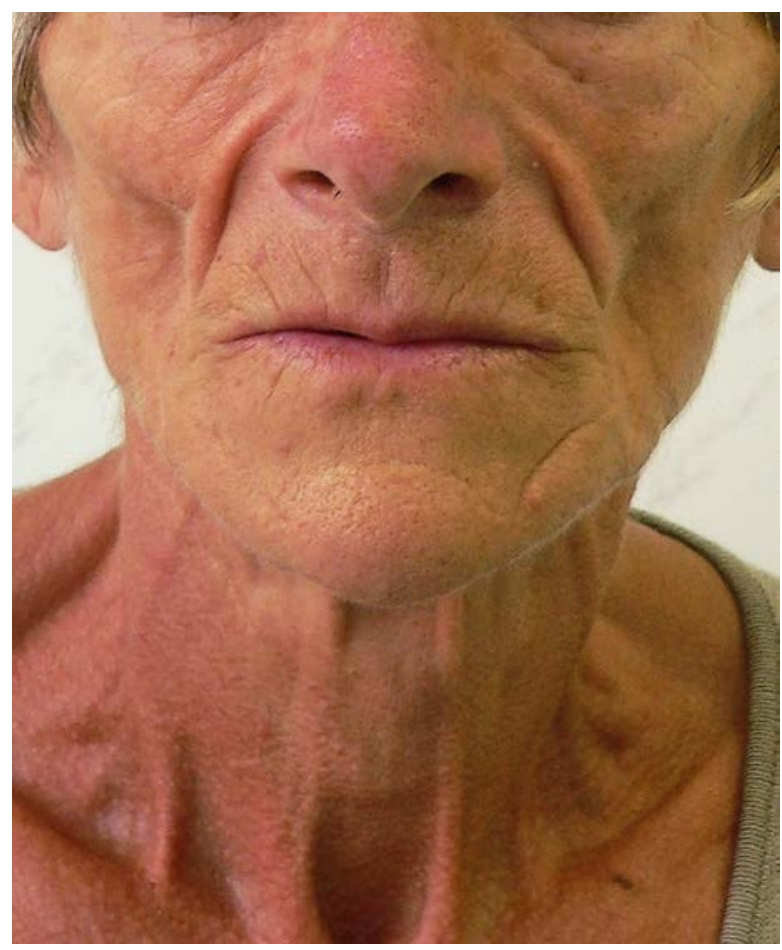

Figure 3. Female with over 20-year duration of HIV infection and 13-year duration of AIDS. Different HAART schemes were undertaken. A complete loss of adipose tissue of both cheeks with strongly protruding facial bone structures 
A high incidence of this disfiguring complication in patients after antiretroviral therapy constitutes a significant therapeutic challenge. People infected with HIV are aware that unnaturally emaciated face may be a visible sign of their health problem and in consequence may lead to social disapproval. The questionnaire survey made by physicians from the University of São Paulo revealed that $84 \%$ of patients with facial lipoatrophy as a side effect of antiretroviral therapy hold a view that the society may recognize them as HIV carriers based on facial deformity [11]. Social isolation and low self-acceptance may cause depression. Decreased quality of life associated with lipodystrophy may lead to rejection of therapy by patients. Therefore, HIV-associated lipodystrophy constitutes a threat to human health and life.

The iatrogenic lipoatrophy may occur as a result of topical or systemic treatment with substances affecting adipose tissue metabolism. Complications due to subcutaneously injected medications like insulin, pethidine, corticosteroids, heparin, growth hormone, erythropoietin and glatiramer acetate, may cause local fat atrophy, but the face is not a common area of injections of these medicaments.

\section{Management of acquired facial lipoatrophy}

Currently, no specific treatment for facial lipoatrophy is known. In the case of lipoatrophy associated with connective tissue disease the point of great importance is early prevention of the atrophy of the adipose tissue by the early diagnosis and management of the disorder in an acute phase to avoid scarring and atrophic lesions. Established changes such as necrosis and subsequent loss of adipose tissue are irreversible. The standard first-line therapy of lupus erythematosus profundus consists of antimalarial agents, such as chloroquine, hydroxychloroquine and quinacrine. Besides having anti-inflammatory and immunosuppressive effects resulting from inhibition of lysosomal proteases, suppression of the mechanisms involved in presentation of the antigens, inhibition of lymphocyte $T$ stimulation and prostaglandin synthesis and blocking a pro-inflammatory cytokine cascade, antimalarials also give partial protection against UV radiation, which may be beneficial for patients with coexisting hypersensitivity to sunlight $[12,13]$. Hydroxychloroquine is recommended as a drug of the first choice, and, if no remission is observed after 2 months of treatment, adding quinacrine or chloroquine can be considered [12, 14]. Excess of the recommended doses and concomitant treatment with chloroquine and hydroxychloroquine should be avoided because of an increased risk of development of ocular complications. Systemic corticosteroid therapy should be administered only in patients with generalized and refractory lesions due to its high toxicity and numerous side effects. A few cases of success ful therapy with thalidomide, dapsone, cyclosporine A, methotrexate, azathioprine and cyclophosphamide have also been described in the literature [15-17].

Establishing an optimal treatment regime for morphea can be difficult due to the limited efficiency of standard medications and not much clinical evidence in support of respective therapies. Topical treatments area therapeutic option for localized scleroderma limited to the skin. Randomized clinical studies of the subcutaneous injection of interferon gamma and oral administration of calcitriol have not proved to be more beneficial than placebo $[18,19]$. A combination of oral prednisone and methotrexate has been proved an effective therapy for the active stage of the disease [20]. In some cases phototherapy with broadband UVA in combination with psoralen (PUVA), narrowband UVB (NB-UVB) or UVA1 may be beneficial [21]. There have been also few reports of successful therapy of morphea with D-penicillamine, cyclosporine, extracorporeal photophoresis and mycophenolate mofetil. A few cases of treatment of deep morphea with abatacept have been also reported, with promising results, as abatacept not only has significantly reduced activity of the disease, but also has softened old and indurated skin lesions [22].

In HIV-positive patients undergoing HAART therapy, the attempt to modify the treatment scheme so as to make it less lipemic (so called "switching" when HIV viremia is undetectable) when lipodystrophy has already occurred rarely leads to noticeable regression of changes [23]. Due to the fact that basically all anti-retroviral drugs may cause lipodystrophy, those responsible for the most intense lipodystrophic symptoms as such should be avoided, especially at the beginning of the treatment. This includes thymidine analogues (D4T and AZT), which belong to a group of nucleoside reverse transcriptase inhibitors (NRTI) [24]. New generation drugs from the aforementioned NRTI group, i.e. tenofovir and abacavir do that to a far lesser extent. Therapy by means of protease inhibitors (PI) also frequently leads to lipodystrophy and metabolic disorders, which is the indication for drug replacement with the other one such as nevirapine (non-nucleoside reverse transcriptase inhibitor - NNRTI) or a new generation PI drug (darunavir or atazanavir) [25]. New generation drugs such as co-receptor inhibitors CCR5 (maraviroc), integrase inhibitors (raltegravir) and a second generation of NNRTI (rilpivirine) seem to carry a substantially lower risk of lipid disorders which implies a lower risk of lipodystrophy [26]. Taking into consideration the fact that the majority of cases of lipodystrophy are accompanied by metabolic disorders, HIV-positive patients should be advised to take up a healthy lifestyle (balanced diet, no cigarettes, and sport activity). If cholesterol and triglycerides levels remain far above the norm, statins or fibrates should be introduced. It is vital to bear in mind important restrictions in relation to which drugs can be jointly applied (drug-drug interactions, especially relates to PIs). The application of met- 
formin as a drug facilitating adipose tissue reduction has an array of limitations including for instance the risk of lactic acidosis, which is especially dangerous in certain HAART therapeutic schemes. The attempts are being made to target lipohypertrophy centrally by means of the recombinant human growth hormone but this may contribute to the further development of peripheral lipoatrophy [27]. The safer bet seems to be an analogue of growth hormone i.e. tesamorelin, which is registered by FDA and if applied subcutaneously is supposed to lead to a reduction in visceral tissue [28].

Facial lipoatrophy remains an enormous therapeutic challenge not only because of difficulties in pharmacotherapy, but also due to progressive esthetic disfigurement of the face that causes social disapproval. Correction of the facial fat loss is crucial for patients with facial lipoatrophy because it helps to reduce stigma and social dysfunction and significantly improves quality of their lives. Unfortunately, there are not many data in the literature concerning the safety and efficacy of particular modalities of correction. Most of them apply to the management of HAART-associated lipoatrophy. This therapy has notably improved survival of HIV-infected patients but on the other hand it has rapidly increased the number of patients with facial lipoatrophy as a complication. There are significantly less reports concerning effects of fat loss correction in patients with connective tissue disorders.

Corrective interventions in facial lipoatrophy include surgical and dermatological procedures such as free dermis-fat grafts, autologous fat transfer and injections of soft tissue fillers. A patient's own fat tissue seems to be ideal tissue filler for it does not cause allergic reactions, is biocompatible and potentially persistent. Free dermis-fat graft is a surgical procedure that requires a hospital stay, poses a risk of overcorrection and is bound to leave scars both in the face and the donor site area. A more commonly-used technique of fat grafting is an autologous fat transfer. The main benefit of this option is that the whole procedure is relatively simple to perform, does not require the patient to stay in the hospital and is followed by a short period of convalescence. This method has been successfully used for volumetric correction of the facial lipoatrophy both HAART-associated and accompanying lupus erythematosus profundus and Parry-Romberg syndrome [29-31]. However, autologous fat transferring in HIV-infected patients may be problematic because of pathological alterations in the metabolism of the adipose tissue, which may lead to either rapid loss or disfiguring hypertrophy of the transferred fat. The biggest drawback of this type of grafting is unpredictability of the final effect due to the possibility of partial reabsorption of the transferred fat over time, which may call for multiple treatment sessions. There have been also observed a few cases of partial necrosis or displacement of the transferred fat, and even one fatal complication caused by a fat embolism in a patient with lupus erythe- matosus profundus, who underwent an autologous fat transfer procedure [32]. Another serious concern is that many people suffering from HAART-related lipodystrophy do not have enough subcutaneous fat on the donor site to make fat tissue transplantation possible.

A relatively new technique of soft tissue augmentation, which has been introduced by the Japanese scientists is called cell-assisted lipotransfer (CAL). In this method standard fat cells derived by liposuction are enriched with adipose derived stem cells, which enhances angiogenesis and reduces the rate of fat reabsorption. The method was originally discovered for breast surgery, but so far it has been applied with success to treatment of facial lipoatrophy associated with 'en coup de sabre' morphea and lupus erythematosus profundus [33].

Synthetic dermal fillers, being widely used for esthetic correction of volume loss in physiological lipoatrophy related to ageing, may also be a beneficial reconstructive method for patients with pathologic lipoatrophy in the course of HIV infection and connective tissue disorders. However, most of those fillers can be used for subdermal volume restoration as an off-label treatment only, excluding poly-L-lactic acid and calcium hydroxyapatite, which have a specific the US Food and Drug Administration (FDA) indication for treatment of HIV-associated lipoatrophy in 2004 and 2006, respectively.

A poly-L-lactic acid is a biocompatible synthetic polymer belonging to a group of aliphatic polyesters. It is not a volumetric filler, but works through the local tissue reaction and stimulates proliferative response of patient's fibroblasts in the place of injection, improving cutaneous thickness. It is immunologically inert thus safe and well tolerated by the patients. Because the material reabsorbs over time, the effect of correction lasts for up to 2 years. In order to accomplish the desired effects, a series of several sessions of injections is required for moderate facial wasting. Poly-L-lactic acid has been used with good results in management of facial lipoatrophy associated with HAART therapy, scleroderma and Parry-Romberg syndrome [34, 35].

Another commonly used dermal filler is calcium hydroxyapatite - a synthetic form of the substance naturally found in the bones and teeth. Microspheres of calcium hydroxyapatite are dispersed in carboxymethyl cellulose carrier gel and are supposed to form a framework for fibroblasts to grow in and proliferate. The volumetric effect is instant and relatively persistent, as the improvement of face contour lasts for up to 18 months. The product is safe and well tolerated by HIV-infected patients and the satisfaction level after correction is high [36]. As for now, there have been no reports concerning the use of calcium hydroxyapatite in volumetric correction of other than HAART therapy-associated pathological facial lipoatrophy.

Hyaluronic acid is one of the main polysaccharides of the extracellular matrix, which adds to volume of the 
skin by promoting keratinocyte proliferation and binding water in the soft tissue. Commercially available products contain partially cross-linked hyaluronic acid derived from bacterial cultures. Experimental studies have shown that, apart from the instant effect of face oval correction, hyaluronic acid fillers induce synthesis of type I collagen which may contribute to prolonged benefits of tissue augmentation [37]. Hyaluronic acid fillers are widely used in esthetic medicine for facial rejuvenation and wrinkle removal and are rarely described as volumizing agents for treatment of facial lipoatrophy connected with fat wasting disorders. However, management of facial volume loss with hyaluronic acid in patients suffering from HAART-related lipoatrophy has provided very good effects, lasting at least 6 months with high patient satisfaction and no serious adverse events. More persistent volumetric effect can be achieved by using fillers with large particle size hyaluronic acid [38, 39]. There have been reports of successful facial augmentation with hyaluronic acid in patients suffering from linear scleroderma of the face [40].

Apart from evident advantages of surgical and dermatological methods for correction of the facial fat loss in patients witch chronic diseases, the above corrective options have limitations, which particularly concern patients suffering from connective tissue diseases. All procedures performed in those patients have, due to antigen stimulation, the potential to increase the risk of reactivation of active, inflammatory lesions, ulceration or even thromboembolic complications. Due to the long-time immunosuppressive treatment of patients with autoimmune disease the process of healing of a surgical wound may be delayed. Thus, it is recommended to perform all surgical interventions exclusively in the controlled, inactive, non-inflammatory phase of disease. A cautious approach of stage-by-stage correction is strongly recommended.

A significant concern and limitation of use of soft tissue fillers in management of facial lipoatrophyis the cost of such treatment. A satisfactory volumetric effect is achieved through a number of sessions. Because soft tissue fillers reabsorb over time, there is a need of constant reapplication of the product to maintain a once achieved result. This makes the therapy costly and out-of-pocket expense for the majority of patients. In few countries a restorative therapy with facial fillers is refunded for the treatment of HAART-related lipodystrophy.

In summary, the relevance of an appearance improvement in patients suffering from serious diseases complicated by facial fat wasting cannot be overestimated. Applying an optimal method of treatment reduces the stigma associated with facial lipodystrophy and significantly improves patients' quality of life. Still, more studies are required to evaluate the safety and efficacy of different therapeutic options in acquired facial lipoatrophy.

\section{Conflict of interest}

The authors declare no conflict of interest.

\section{References}

1. Herranz P, de Lucas R, Perez-Espana L, et al. Lipodystrophy syndromes. Dermatol Clin 2008; 26: 569-78.

2. Krysiak R, Rudzki H, Okopień B. Lipodystrofia: nowe spojrzenie na starą chorobę. Przegl Lek 2012; 69: 149-56.

3. Ascher B, Coleman S, Alster T, et al. Full scope of effect of facial lipoatrophy: a framework of disease understanding. Dermatol Surg 2006; 32: 1058-69.

4. Fenske NA, Lober CW. Structural and functional changes of normal aging skin. J Am Acad Dermatol 1986; 15: 571-85.

5. Arnold HL. Lupus erythematosus profundus: commentary and report on four more cases. Arch Dermatol 1956; 73: 15-33.

6. Peterson LS, Nelson AM, Su WP. Classification of morphea (localized scleroderma). Mayo Clin Proc 1995; 70: 1068-76.

7. Fett N, Werth VP. Update on morphea: Part I. Epidemiology, clinical presentation and pathogenesis. J Am Acad Dermatol 2011; 64: 217-28.

8. Carr A, Samaras K, Burton S, et al. A syndrome of peripheral lipodystrophy, hyperlipidaemia and insulin resistance in patients receiving HIV protease inhibitors. AIDS 1998; 12: 51-8.

9. Carr A, Samaras K, Thorisdottir A, et al. Diagnosis, prediction and natural course of HIV-1 protease-inhibitor-associated lipodystrophy, hyperlipidaemia, and diabetes mellitus: a cohort study. Lancet 1999; 353: 2093-9.

10. James J, Carruthers A, Carruthers J. HIV-associated facial lipoatrophy. Dermatol Surg 2002; 28: 979-86.

11. Sanches RS, Mill J, Machado AA, et al. Facial lipoatrophy: appearances are not deceiving. J Assoc Nurses AIDS Care 2009; 20: 169-75.

12. Wozniacka A, McCauliffe DP. Optimal use of antimalarials in treating cutaneous lupus erythematosus. Am I Clin Dermatol 2005; 6: 1-11.

13. Kalia S, Dutz JP. New concepts of antimalarial use and mode of action in dermatology. Dermatol Ther 2007; 20: 160-74.

14. Kiedrowicz M, Kacalak-Rzepka A, Bielecka-Grzela S, et al. Antimalarial drugs in contemporary dermatologic therapy. Ann Acad Med Stetin 2011; 57: 38-44.

15. Wientert S, Gadola S, Hunziker T. Facets of lupus erythematosus: panniculitis responding to thalidomide. J Dtsch Dermatol Ges 2008; 6: 214-6.

16. Yamada Y, Dekio S, Jidoi J, et al. Lupus erythematosus profundus: report of a case treated with dapsone. J Dermatol 1989; 16: 379-82.

17. Grossberg E, Scherschun L, Fivenson DP. Lupus profundus: not a benign disease. Lupus 2001; 10: 514-6.

18. Hunzelmann N, Anders S, Fierlbeck G, et al. Double-blind, placebo-controlled study of intralesional interferon gamma for the treatment of localized scleroderma. J Am Acad Dermatol 1997; 36: 433-5.

19. Hulshof MM, BouvesBavinck JN, Bergman W, et al. Double-blind, placebo-controlled study of oral calcitriol for the treatment of localized and systemic scleroderma. J Am Acad Dermatol 2000; 43: 1017-23.

20. Zulian F, Martini G, Vallongo C, et al. Methotrexate treatment in juvenile localized scleroderma: a randomized, double-blind, placebo-controlled trial. Arthritis Rheum 2011; 63: 1998-2006.

21. Kreuter A, Hyun J, Stucker M, et al. A randomized controlled study of low-dose UVA1, medium-dose UVA1 and narrow- 
band UVB phototherapy in the treatment of localized scleroderma. J Am Acad Dermatol 2006; 54: 440-7.

22. Stausbol-Gron B, Olesen AB, Deleuran B, et al. Abatacept is a promising treatment for patients with disseminated morphea profunda: presentation of two cases. Acta Derm Venereol 2011; 91: 686-8.

23. Grunfeld C, Saag M, Cofrancesco J, et al. Study of Fat Redistribution and Metabolic Change in HIV Infection (FRAM). Regional adipose tissue measured by MRI over 5 years in HIV-infected and control participants indicates persistence of HIV-associated lipoatrophy. AIDS 2010; 24: 1717-26.

24. Haubrich RH, Riddler S, Dirienzo G, et al. Metabolic outcomes in a randomized trial of nucleoside, non-nucleoside and protease inhibitor sparing regimens for initial HIV treatment. AIDS 2009; 23: 1109-18.

25. Stanley TL, Joy T, Hadigan CM, et al. Effects of switching from lopinavir/ritonavir to atazanavir/ritonavir on muscle glucose uptake and visceral fat in HIV-infected patients. AIDS 2009; 23: 1349-57.

26. Nozza S, Galli L, Visco F, et al. Raltegravir, maraviroc, etravirine: an effective protease inhibitor and nucleoside reverse transcriptase inhibitor-sparing regimen for salvage therapy in HIV-infected patients with triple-class experience. AIDS 2010; 24: 924-8

27. Tai VW, Schambelan M, Algren $\mathrm{H}$, et al. Effects of recombinant human growth hormone on fat distribution in patients with human immunodeficiency virus-associated wasting. Clin Infect Dis 2002; 35: 1258-62.

28. Falutz J, Allas S, Blot K, et al. Metabolic effects of a growth hormone releasing factor in patients with HIV. N Engl I Med 2007; 357: 2359-70.

29. Valdatta L, Cherubino M, Tamborini F, et al. A case of facial lipoatrophy secondary to lupus profundus managed with lipofilling technique. Case Rep Dermatol Med 2012; 2012: 720518.

30. Uzzan C, Boccara D, Lacheré A, et al. Treatment of facial lipoatrophy by lipofilling in HIV infected patients: retrospective study on 317 patients on 9 years. Ann Chir Plast Esthet 2012; 57: 210-6.

31. Sterodimas A, Huanquipaco JC, de Souza Filho S, et al. Autologous fat transplantation for the treatment of Parry-Romberg syndrome. J Plast Reconstr Aesthet Surg 2009; 62: 424-6.

32. Gleeson CM, Lucas S, Langrish CJ, et al. Acute fatal fat tissue embolism after autologous fat transfer in a patient with lupus profundus. Dermatol Surg 2011; 37: 111-5.

33. Yoshimura K, Sato K, Aoi N, et al. Cell-assisted lipotransfer for facial lipoatrophy: efficacy of clinical use of adipose-derived stem cells. Dermatol Surg 2008; 34: 1178-85.

34. Lafaurie M, Dolivo M, Porcher R, et al. Treatment of facial lipoatrophy with intradermal injections of polylactic acid in HIV-infected patients. J Acquir Immune Defic Syndr 2005; 38: 393-8.

35. Onesti MG, Troccola A, Scuderi N. Volumetric correction using poly-L-lactic acid in facial asymmetry: Parry Romberg syndrome and scleroderma. Dermatol Surg 2009; 35: 1368-75.

36. Silvers SL, Eviatar JA, Echavez MI, et al. Prospective, open-label, 18-month trial of calcium hydroxylapatite (Radiesse) for facial soft-tissue augmentation in patients with human immunodeficiency virus-associated lipoatrophy: one-year durability. Plast Reconstr Surg 2006; 118 (3 Suppl): 34S-45S.

37. Wang F, Garza LA, Kang S, et al. In vivo stimulation of de novo collagen production caused by cross-linked hyaluronic acid dermal filler injections in photodamaged human skin. Arch Dermatol 2007; 143: 155-63.
38. Gooderham M, Solish N. Use of hyaluronic acid for soft tissue augmentation of HIV-associated facial lipodystrophy. Dermatol Surg 2005; 31: 104-8.

39. Pignatti M, Pedone A, Baccarani A, et al. High-density hyaluronic acid for the treatment of HIV-related facial lipoatrophy. Aesthetic Plast Surg 2012; 36: 180-5.

40. Choksi MD, Jeffreys S, Orringer MD. Linear morphea-induced atrophy treated with hyaluronic acid filler injections. Dermatol Surg 2011; 37: 880-8. 